\title{
Ecología reproductiva de Geobatrachus walkeri (Anura: Srabomantidae)
}

\author{
Vanesa Pacheco-Florez ${ }^{1} \&$ Martha P. Ramírez-Pinilla ${ }^{2 *}$ \\ 1. Universidad del Magdalena, Santa Marta, Colombia; tatianam86@gmail.com, vanesasofi@gmail.com \\ 2. Laboratorio de Biología Reproductiva de Vertebrados, Universidad Industrial de Santander, Bucaramanga, Colombia; \\ mpramir@uis.edu.co,mpramir@gmail.com \\ * Correspondencia
}

Recibido 10-IV-2013. Corregido 20-IX-2013. Aceptado 30-X-2013.

\begin{abstract}
Reproductive ecology of Geobatrachus walkeri (Anura: Strabomantidae). Geobatrachus walkeri is a frog that belongs to a monotypic genus and is endemic to the Sierra Nevada de Santa Marta, Colombia. The species inhabits different microhabitats between 2000 to $3500 \mathrm{~m}$ altitude, including the leaf litter of a pine plantation. To understand its reproductive ecology, we conducted eight frog samplings, covering the rainy and dry seasons, and two habitat types (secondary native forest and pine plantation) during 2010-2011. For this study, we also included data obtained from five previous similar samplings undertaken during 2008-2009. The pine leaf litter was the main microhabitat where frogs were found; we heard choruses of six-ten calling males during all sampled months, and observed the frogs having diurnal and nocturnal activity. Regardless of the year of study, the population consisted of neonates recruited several times of the year, a large number of juveniles with a wide range of body sizes, and fewer adults with a narrower range of body size. The histological analyses of the gonads showed that the size at maturity was near $18 \mathrm{~mm}$ SVL for males and females, and those adult males and females were reproductive active during all sampling months, suggesting a continuous reproductive activity pattern. However, during the dry season, the seminiferous tubules showed a drastically diminished spermatic epithelium although containing abundant luminal spermatozoa, which suggest a reduction in the sperm production at the end of this season. Similarly, frogs of all age categories were significantly more abundant during the early dry season, whereas were significantly less abundant with the advancement of the dryness suggesting that the intensity of the dry season could temporally stop the activity and reproduction of this population. Rev. Biol. Trop. 62 (1): 183-199. Epub 2014 March 01.
\end{abstract}

Key words: reproductive activity, Sierra Nevada de Santa Marta, tropical seasonality, mountain leaf litter frog, gonad histology.

Los anuros tienen diferentes formas de independencia del agua y de acuerdo con ellas se ha determinado la existencia de varias estrategias o modos reproductivos (Bolaños, 2003; Duellman \& Trueb, 1994). Con relación al tiempo de actividad reproductiva se han descrito asimismo diferentes patrones reproductivos: explosivos, prolongados y permanentes (Duellman \& Trueb, 1994; Pough et al., 2004). Tanto los modos reproductivos como estos patrones temporales de actividad reproductiva se ven influenciados por factores externos como la precipitación y la temperatura ambiente (Donnelly
\& Guyer, 1994), pero además cada especie presenta un modo y un patrón reproductivo en respuesta a las condiciones de su entorno y a su historia evolutiva (Crump, 1982).

En las especies de desarrollo directo de Terrarana se esperaría que la reproducción fuese continua debido a que estas ranas son en cierta forma independientes de los cuerpos o cursos de agua; este modo reproductivo le otorga a este clado ventajas para la ocupación y explotación de ambientes menos asociados al agua. Sin embargo, los huevos son usualmente puestos entre la hojarasca requiriendo entonces 
humedades relativas altas para su desarrollo; por lo tanto, dependiendo de los lugares de oviposición y de las condiciones de humedad del ambiente, los huevos podrían estar expuestos a la deshidratación (Marsh \& Pearman, 1997; Tocher, Gascon, \& Zimmerman, 1997). En ambientes fuertemente estacionales del Neotrópico, una estación seca prolongada o extrema podría afectar especialmente a la herpetofauna de hojarasca al restringir la disponibilidad de microhábitats con una humedad relativa suficiente para la oviposicición y desarrollo de las posturas, o indirectamente al disminuir la productividad primaria y la descomposición (Scott, 1976). Los estudios en varias especies y poblaciones de especies de Terrarana han efectivamente mostrado actividad reproductiva continua (p.ej. Pristimantis croceoinguinis, P. lanthanites, P. variabilis, Crump, 1974; Craugastor bransfordii, Donnelly, 1999; E. eugeniae Guayasamin, Bonaccorso, Menéndez, \& Bustamante, 2004; Eleutherodactylus johnstonei, Ortega, Serrano \& Ramírez-Pinilla, 2005); sin embargo, para algunas de ellas la producción de posturas y aparición de juveniles ocurre principalmente en los meses más secos (p. ej. Strabomantis biporcatus, Craugastor mimus, C. talamancae, C. fitzingeri, Watling \& Donnelly, 2002; E. coqui, Towsend \& Stuart, 1994), o en los meses de la estación de lluvias (p. ej. E. johnstonei, Bourne, 1997; Ovaska, 1991; E. cuneatus Fong, Hero, Viña \& Bignotte-Giró, 2010). Algunas especies pueden tener una actividad reproductiva marcadamente estacional relacionada con pequeños cambios en la temperatura (p. ej. E. cooki cesa la oviposición en la temporada fría, Rogowitz, Candelaria, Denizard,\& Meléndez, 2001) o de precipitación (p.ej. Haddadus binotatus Canedo \& Rickli, 2006).

Geobatrachus walkeri es una especie que pertenece a un género monotípico y endémico de la Sierra Nevada de Santa Marta; se asemeja a las ranas del género Pristimantis en su forma de vida, morfología y porque presenta desarrollo directo, forma parte del clado Terrarana y dentro de él ha sido ubicado provisionalmente dentro de la familia Strabomantidae y subfamilia Strabomantinae (Hedges, Duellman \& Heinicke, 2008). Inicialmente su distribución se restringía al sector de San Lorenzo en hábitats de bosque subtropical y tropical húmedo montano, y en plantaciones de pino de esta zona (Ardila-Robayo, 1979; Ramírez-Pinilla, Osorno-Muñoz, Rueda, Ardila-Robayo \& Amézquita, 2004), pero recientemente se registró a $3500 \mathrm{~m}$ de altura en ecosistema páramo (RuedaSolano \& Vargas-Salinas, 2010). La especie ha sido categorizada en la lista roja UICN como en peligro (EN B1 ab(iii), Ramírez-Pinilla et al., 2004; UICN, 2009) debido a que su área de distribución es menor a $5000 \mathrm{~km}^{2}$, sus poblaciones se encuentran establecidas en menos de cinco localidades, y persiste una disminución en la calidad y extensión de su hábitat.

Respecto de su historia natural se han realizado estudios comparativos de uso de microhábitat y abundancia de esta especie entre el bosque nativo y las plantaciones de pino en el sector de San Lorenzo (Martínez-Baños, Pacheco-Florez \& Ramírez-Pinilla, 2011). De manera interesante, los autores determinaron que la mayor abundancia de individuos se encontraba en las plantaciones de pino y durante la época de lluvia, ocupando específicamente la hojarasca de pino en donde registraron posturas. Adicionalmente registraron machos cantando en coros durante todo el año por lo que sugirieron una actividad reproductiva potencialmente continua con un posible pico reproductivo durante la estación de lluvias.

En este estudio se continúa con el trabajo iniciado sobre la biología de esta interesante especie con la descripción de aspectos de su ecología reproductiva, específicamente con los objetivos de identificar el patrón de actividad reproductiva de G. walkeri (explosivo, prolongado o permanente), establecer la influencia de diferentes variables ambientales sobre este patrón y describir características importantes asociadas a los aspectos reproductivos. 


\section{MATERIALES Y MÉTODOS}

El área de estudio se localiza en el sector de San Lorenzo, en el flanco nor-occidental de la Sierra Nevada de Santa Marta, departamento del Magdalena, Colombia $\left(11^{\circ} 6^{\prime} \mathrm{N}-74^{\circ} 3^{\prime} \mathrm{W}\right)$. Específicamente se estudió la población que se distribuye entre los 2300 y $2500 \mathrm{~m}$ de altitud y cuyas características de hábitat y microhábitat fueron descritas en el estudio de MartínezBaños et al. (2011). La precipitación media anual de la zona de estudio es de $2622 \mathrm{~mm}$, y la temperatura media anual de $13.6^{\circ} \mathrm{C}$. Esta área registra un régimen de precipitación unimodal biestacional presentando la estación seca entre los meses de diciembre a marzo y la estación de lluvias de abril a noviembre (Fig. 1).

La actividad reproductiva de $G$. walkeri se determinó a través de muestreos por cuadrantes y la técnica VES (búsqueda por encuentro casual, Crump \& Scott, 1994). Se realizaron ocho muestreos entre los meses de mayo 2011 a enero 2012 abarcando las estaciones de lluvia (mayo a noviembre) y sequía (diciembre y enero) y los dos tipos de hábitats allí encontrados (bosque nativo secundario y plantación de pino). Durante todo el periodo de muestreo se revisó cada uno de los 64 cuadrantes de $4 \times 4 \mathrm{~m}$ establecidos al azar en ambos hábitats, 32 por cada tipo de hábitat. Se revisaron seis cuadrantes elegidos aleatoriamente por día de muestreo, tres en cada zona de vegetación (plantación de pino y bosque nativo), y rotando durante cada salida de campo la hora de muestreo de manera que se cubrieran cuadrantes durante el día y la noche. Cada uno de los cuadrantes era examinado exhaustivamente por un máximo de dos horas por una persona. En cada cuadrante se buscaron sitios de postura y la presencia de las ranas; se registró para cada rana la hora del avistamiento, si se encontraba activa (p.ej. forrajeando, desplazándose) o inactiva ( $\sin$ movimiento dentro de refugios) y si había algún tipo de comportamiento reproductivo (canto, amplexo), su tamaño (longitud rostro-cloacal, LRC, con un calibrador Vernier $\pm 0.02 \mathrm{~mm}$ de precisión) y peso (pesola ${ }^{\circledR}, \pm 0.1 \mathrm{~g}$ de precisión), y presencia de huevos abdominales por palpación. Para estos animales no se determinó por observación macroscópica el sexo a menos que se palparan huevos abdominales evidentes o que se evidenciara el comportamiento de canto. Siempre que se registraron animales en un cuadrante se tomaron datos de variables ambientales como humedad relativa y temperatura del suelo (termohigrómetro portátil HI 856). La cobertura vegetal se determinó siguiendo el mismo método descrito en Martínez-Baños et al. (2011). Para determinar si la cobertura vegetal, la temperatura y la humedad relativa de los microhábitats donde se encontraron las ranas es diferente entre meses de muestreo y periodos climáticos (i.e. lluvioso y seco), se emplearon pruebas de Kruskal-Wallis. El muestreo por VES se realizó durante dos horas de día y dos horas de noche, durante dos días en cada salida. A través de esta metodología se buscó realizar registros complementarios de actividad y comportamientos reproductivos.

Se registraron las llamadas de cinco individuos con una grabadora de bolsillo (Panasonic RR-QR270), los cuales fueron posteriormente medidos (LRC) y liberados. Las propiedades espectrales y temporales de las llamadas fueron analizadas en tres llamadas por cada macho utilizando el software Raven (Charif, Ponirakis \& Krein, 2008). Cada llamada fue descrita según parámetros temporales (duración de la llamada, número de notas, intervalo entre llamadas, intervalo entre notas) y parámetros espectrales (frecuencia dominante, baja y alta de cada nota). Las frecuencias baja y alta fueron medidas a $20 \mathrm{~dB}$ (re $20 \mu \mathrm{Pa}$ ) por debajo de la intensidad pico, donde la señal es claramente distinguible del ruido de fondo de las grabaciones. Para la medición de los parámetros acústicos, nos basamos en la terminología y los procedimientos descritos por Cocroft \& Ryan (1995). Cada parámetro fue promediado para representar la unidad estadística de análisis más pequeña.

Con base en los datos totales de los animales observados en cada muestreo, se graficó el tamaño de los individuos encontrados (LRC) con respecto al tiempo de muestreo y 

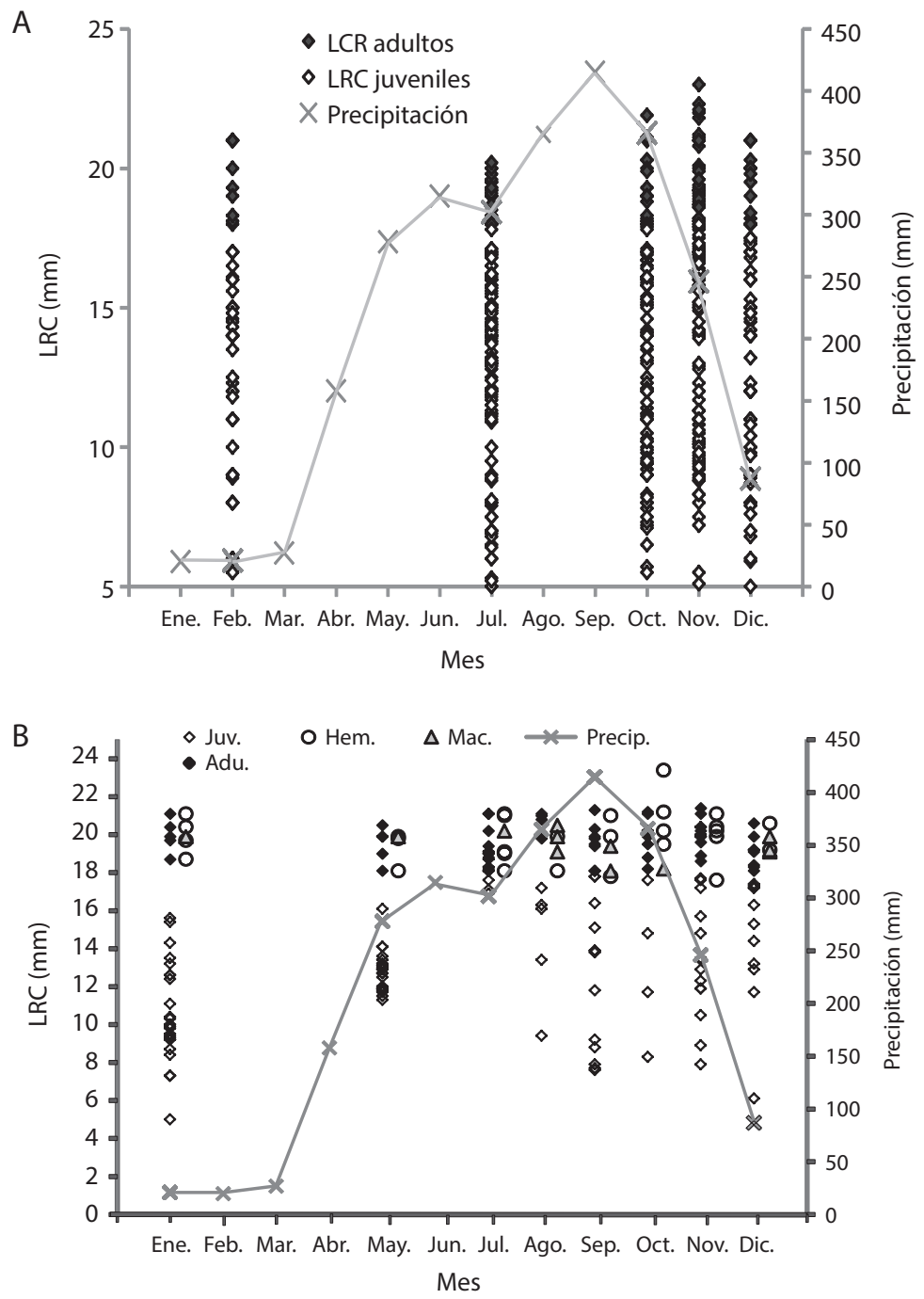

Fig. 1. Composición de la población, categoría de edad-talla en (A) 2008-2009, (B) 2010-2011. En el muestreo de 2010-2011 (Figura 1B) se observan las hembras adultas y los machos adultos derivados del análisis histológico de los tractos. También se observa el promedio mensual multianual de precipitación como una línea gris.

Fig. 1. Population composition of Geobatrachus walkeri (age category-size) of the study years (A) 2008-2009 and (B) 20102011. In the figure 1B from 2010-2011 are observed the adult females and the adult males derived from the histological analyses. The multiannual monthly mean of rains is also observed as a grey line.

en relación con la precipitación mensual. Para determinar si existieron diferencias significativas en la distribución temporal del número total de ranas registradas, de adultos y juveniles entre muestreos se usaron pruebas de bondad y ajuste. Para determinar si existieron diferencias significativas en el tamaño medio de todos los individuos observados, de los adultos y de los juveniles en el tiempo y entre estaciones se realizaron prueba de ANOVA o Kruskal Wallis (según corresponda) seguidas de pruebas $a$ posteriori. A manera comparativa y complementaria se tomaron los datos originales de los muestreos realizados en 2008-2009 para la misma población y parcialmente publicados en el estudio de Martínez-Baños et al. (2011). 
Para ese año se hicieron muestreos según la metodología consignada en ese estudio en los meses de julio, octubre, noviembre, diciembre y enero. Aunque el esfuerzo de captura en ese año fue aproximadamente el doble del presente estudio, queremos determinar si en los dos años muestreados hubo diferencias significativas entre meses y estaciones, en el número y tamaño corporal de los individuos en general, en adultos y juveniles, y en los meses de aparición de neonatos. Por lo tanto, se realizaron los mismos análisis previamente descritos para los especímenes de 2010-2011. Para ambos casos, si se encontraran diferencias significativas entre muestreos, estaciones o en meses particulares, especialmente para los individuos juveniles, se podría sugerir una distribución temporal particular de tamaños asociada con una actividad reproductiva estacional marcada.

Con los datos de los LRC de las ranas que se encontraron activas y las horas en las que se registraron en los muestreos de 2008-2009 y 2010-2011 y se hizo una gráfica para visualizar el patrón de actividad diario en relación con el tamaño corporal. Se ha sugerido que es común que los individuos juveniles de las especies de ranas del clado Terrarana tengan predominantemente una actividad diurna mientras que los adultos son activos en la noche (Donnelly, 1994 p. ej. en P. fenestratus Menin, Waldez, \& Lima, 2008; Pristimantis thectopternus Rincón-Castro \& Castro Herrera, 1998) como una manera de evitar la depredación, o la competencia por los recursos, o porque como en los sapos los juveniles son heliotérmicos mientras los adultos no (Wells, 2007; Urbina \& Galeano, 2009). La gráfica permitirá visualizar si este patrón sugerido es cierto para la población de esta especie.

Durante los muestreos en 2010-2011 se recolectaron 42 ranas para verificar el tamaño de madurez sexual en ambos sexos y la condición reproductiva de los adultos, aproximadamente cinco individuos por mes de muestreo. Los datos derivados de la determinación del tamaño de madurez sexual permitieron separar las categorías de adultos y juveniles usados para los análisis de variación temporal y actividad descritos anteriormente. Las ranas recolectadas fueron eutanizadas en cloretona al $10 \%$, fijadas en formol al $10 \%$ y posteriormente se almacenaron para su traslado en etanol al $70 \%$. Los especímenes fueron llevados al Laboratorio de Biología Reproductiva de Vertebrados de la Universidad Industrial de Santander, Bucaramanga, Colombia, en donde se estudiaron los tractos reproductivos. Para ello se hizo una disección abdominal para extraer los tractos reproductivos completos. Una vez retirados los órganos reproductivos, se procedió a conservar en etanol al $70 \%$ los tractos digestivos para futuros estudios de la dieta de esta población.

Bajo estereoscopio se tomaron medidas de largo y ancho de los testículos en machos, y diámetro mayor de los ovarios y folículo más grande en las hembras, utilizando un calibrador Vernier (precisión de $0.2 \mathrm{~mm}$ ). Se calculó el volumen de los dos testículos de cada individuo por medio de la fórmula del esferoide $\left[\mathrm{V}=4 / 3\left(\Pi \mathrm{a}^{2} \mathrm{~b}\right)\right.$, Wiederhecker, Pinto $\&$ Colli, 2002], posteriormente se comparó el volumen entre ambos testículos mediante una prueba de $t$ student de dos colas, para continuar los análisis utilizando indiscriminadamente o no, el testículo izquierdo o derecho. Según las características macroscópicas de las gónadas de las ranas, las hembras se clasificaron en: 1) inmaduras (folículos ováricos no yemados, granulares - menos de $0.5 \mathrm{~mm}$ de diámetro- y oviductos delgados y no convolutos), 2) no reproductivas (folículos ováricos mayores a $0.5 \mathrm{~mm}$ de diámetro pero no yemados y oviductos no convolutos, algo engrosados), 3) reproductivas (con folículos ováricos yemados $\mathrm{y}$ oviductos convolutos, gruesos y turgentes), 4) grávidas (con oviductos conteniendo huevos o huevos libres abdominales), o 5) post-grávidas (con folículos no yemados y oviductos convolutos, muy gruesos y flácidos). En caso de encontrar hembras en diferentes estados reproductivos en los muestreos se utilizan pruebas $\mathrm{G}$ de razón de log verosimilitud para determinar si hay diferencias significativas en la ocurrencia de hembras en cada estado reproductivo entre muestreos. 
Para el análisis del estado reproductivo en los machos y etapa de desarrollo folicular en las hembras, se realizaron análisis histológicos de las gónadas mediante histotecnia corriente. Los tejidos fueron deshidratados en una serie ascendente de soluciones de etanol y posteriormente embebidos en parafina; los cortes de $5 \mu \mathrm{m}$ de espesor fueron realizados usando micrótomo de rotación corriente y se tiñeron con hematoxilina- eosina. Con base en estos cortes se registró la etapa de desarrollo en la cual se encontraron los folículos ováricos y se contó el número de los folículos ováricos en estado más avanzado de desarrollo. La identificación de las etapas de desarrollo de los folículos ováricos se basó en Prado, Abdalla, Silva \& Zina (2004).

Espermatogonias, espermatocitos, espermátidas y espermatozoides fueron identificados para cada sección testicular de cada individuo. Los machos con espermatozoides libres en el lumen de los túbulos seminíferos fueron considerados como reproductivos. La talla mínima de madurez sexual se determinó según las características reproductivas observadas en los machos y hembras colectados de manera que se consideró el tamaño (LRC) de la hembra más pequeña que fuera reproductiva, grávida o postgrávida, y el tamaño (LRC) del macho más pequeño reproductivo. Las ranas con menores LRC fueron clasificadas como juveniles.

Para establecer si había dimorfismo sexual por tamaño corporal (LRC y peso) se utilizaron los registros de las 40 ranas adultas recolectadas porque de ellas se tenía plena confirmación de su sexo y estado de madurez. Para ello, se realizaron pruebas de U-Mann Whitney. Para determinar si el tamaño corporal de las hembras estaba relacionado con el número y diámetro mayor de los folículos yemados se utilizaron pruebas de correlación de Pearson.

El tamaño de las gónadas (diámetro folicular en hembras y el volumen testicular en machos) puede estar influenciado por el tamaño corporal, de manera que para determinar si la relación entre el tamaño corporal (LRC) y el tamaño de las gónadas en cada sexo era significativa, se utilizó un análisis de regresión. En el caso de que esta relación fuera significativa y para determinar si había diferencias significativas en el tamaño ajustado de las gónadas entre meses y entre las estaciones seca y de lluvias se utilizaron análisis de covarianza ANCOVA, en donde la variable LRC es la covariable que controló la variación asociada al tamaño del cuerpo. En el caso de que esta relación no fuera significativa se utilizó ANOVA con los datos originales de tamaño gonadal.

La asociación entre el tamaño promedio de las gónadas en ambos sexos y por estación con respecto de la media de precipitación fue determinada utilizando un análisis de correlación de Spearman ya que los datos de precipitación no cumplen los requerimientos para análisis paramétricos. Previo a los análisis estadísticos se determinó la normalidad de los datos y la homocedasticidad mediante las pruebas de Shapiro-Wilk and Levene, respectivamente. Todos los análisis estadísticos siguen a Zar (1999) y el valor de significancia $p$ se estableció en 0.05 .

\section{RESULTADOS}

En total se registraron 157 ranas durante todo el muestreo: 94 juveniles y 63 adultos. Sólo se lograron observar cuatro ranas en el bosque nativo, a pesar de emplear un mismo esfuerzo de muestreo para ambas clases de ambientes (bosque nativo y plantación de pino). Para los muestreos realizados en 2008-2009 se avistaron 522 ranas en total, de las cuales 386 fueron juveniles y 116 fueron adultos. El número de ranas en el bosque nativo $(\mathrm{N}=140)$ fue mucho menor que en la plantación de pinos $(n=382)$. Para los dos años de muestreos el principal microhábitat donde se encontraron las ranas fue entre la hojarasca de pino, ya sea directamente entre las acículas de pino o bajo troncos o piedras que están sobre esta hojarasca. Allí se escucharon grupos de coros de seis a 10 individuos durante todos los muestreos, incluyendo los meses de más baja precipitación (diciembre y enero).

Se encontraron juveniles y adultos durante todos los meses muestreados. En las figuras 1 (A y B) se puede visualizar la información de 
número y tamaño de todos los individuos discriminados por adultos y juveniles encontrados en los dos años de muestreo, en relación con la precipitación media mensual multianual para la zona de estudio. En el cuadro 1, se resume la variación encontrada para los dos años de muestreos entre meses y estaciones para el número y LRC de los individuos total avistados y de los adultos y juveniles separadamente, al igual que se muestran los meses en los que se encontraron ranitas recién eclosionadas (menos de 6mm de LRC). Para 2008-2009 el número de ranas varió significativamente entre los meses, siendo menor en el mes de febrero, mes que no fue muestreado posteriormente en 2011; en este último año también el número de ranas varió significativamente pero mostró que enero fue el mes que más ranas aportó, específicamente en juveniles, enero sin embargo, no fue muestreado en 2009. Mientras en los meses de lluvias en los dos años se encuentran los mayores promedios de tamaño corporal, en el mes seco de enero 2011 se encontraron los menores tamaños corporales (Media=10.3, DE=2.6mm) indicando que los juveniles más pequeños aparecen en la población en el mes de enero. Sin embargo, los neonatos aparecen registrados en todos los meses en el muestreo de 2008-2009 y en los meses de la estación seca en 2010-2011. Por lo tanto, independientemente del año de estudio, se puede observar que la población de $G$. walkeri consiste de neonatos que son reclutados varias veces al año, un gran número de juveniles con un amplio rango de tamaños corporales y un menor número de adultos con un rango de tamaño corporal más estrecho.

Del total de individuos observados en 2010-2011 (157), 69 se encontraron activos forrajeando, caminando entre la hojarasca o cantando, mientras que los restantes 88

\section{CUADRO 1}

Comparación de la variación entre meses y entre estaciones de la cantidad y tamaño promedio de ranas adultas y juveniles de Geobatrachus walkeri en las dos temporadas de estudio

TABLE 1

Comparison of the intra-month and intra-season variation in number and mean size of adult and juvenile Geobatrachus walkeri frogs in the two study years

\begin{tabular}{|c|c|c|c|c|c|c|}
\hline \multirow{2}{*}{ Variables } & \multicolumn{2}{|c|}{$2008-2009$} & \multicolumn{3}{|c|}{ 2010-2011 } & \multirow{2}{*}{ Variación } \\
\hline & Entre meses & Entre estaciones* & Variación & Entre meses & Entre estaciones* & \\
\hline Número total de ranas & $\begin{array}{l}X^{2}=28, g l=4 \\
p<0.001\end{array}$ & & $\begin{array}{l}\text { Febrero menos } \\
\text { ranas }\end{array}$ & $\begin{array}{l}X^{2}=20, g l=7 \\
p=0.05\end{array}$ & & Enero más ranas \\
\hline Número total de adultos & $\begin{array}{l}\mathrm{X}^{2}=20, \mathrm{gl}=4, \\
\mathrm{p}<\mathbf{0 . 0 0 1}\end{array}$ & & $\begin{array}{l}\text { Febrero menos } \\
\text { adultos }\end{array}$ & $\begin{array}{l}X^{2}=8.25, g l=7 \\
p=0.31\end{array}$ & & \\
\hline Número total de juveniles & $\begin{array}{l}X^{2}=21.8, g l=4 \\
p=0.0002\end{array}$ & & $\begin{array}{l}\text { Febrero menos } \\
\text { juveniles }\end{array}$ & $\begin{array}{l}X^{2}=41.83, g l=7, \\
\mathrm{p}<0.001\end{array}$ & & $\begin{array}{l}\text { Enero más } \\
\text { juveniles }\end{array}$ \\
\hline LRC de todas las ranas & $\mathrm{H}=10.8, \mathrm{p}=\mathbf{0 . 0 3}$ & $\mathrm{H}=3.2, \mathrm{p}=0.08$ & $\begin{array}{l}\text { Noviembre } \\
\text { mayor LRC }\end{array}$ & $\begin{array}{l}H=36.6 \\
p<0.001\end{array}$ & $\mathrm{H}=13, \mathrm{p}<\mathbf{0 . 0 0 1}$ & $\begin{array}{l}\text { Enero y sequía } \\
\text { menor LRC }\end{array}$ \\
\hline LRC de las ranas adultas & $\mathrm{H}=6.4, \mathrm{p}=0.17$ & $\mathrm{H}=0.3, \mathrm{p}=0.6$ & & $\begin{array}{l}H=14.8 \\
p=\mathbf{0 . 0 4}\end{array}$ & $\mathrm{H}=0.37, \mathrm{p}=0.54$ & $\begin{array}{l}\text { Agosto mayor } \\
\text { LRC }\end{array}$ \\
\hline LRC de los juveniles & $\mathrm{H}=5.1, \mathrm{p}=0.3$ & $\mathrm{H}=2.5, \mathrm{p}=0.11$ & & $\begin{array}{l}H=29.3 \\
p<0.001\end{array}$ & $\mathrm{H}=3.3, \mathrm{p}=0.07$ & $\begin{array}{l}\text { Enero menor y } \\
\text { Julio mayor LRC }\end{array}$ \\
\hline $\begin{array}{l}\text { Neonatos ( }>6 \mathrm{~mm} \text { de LRC) } \\
\text { en los meses }\end{array}$ & & eb, Jul, Oct, Nov, I & & & Dic, Ene & \\
\hline
\end{tabular}

* La variación entre estaciones no se determinó para estas variables porque el esfuerzo de muestreo no fue similar entre estaciones (hay más meses de lluvias que meses secos). 
individuos fueron encontrados inactivos inmersos dentro de la hojarasca mientras se revisaban los cuadrantes. Sumando los datos de ranas que se encontraron realizando alguna actividad que las hizo evidentes a los observadores para 20082009, se puede ver que la distribución horaria de la actividad de estas ranas es amplia (se observó actividad diurna y nocturna). Durante el día se concentró especialmente en las horas de la mañana y al finalizar de la tarde y aunque se observaron activos durante toda la noche, la mayor cantidad de ranas activas se encontraron en las primeras horas de la noche (Fig. 2). Las ranas de todos los tamaños estuvieron activas durante todo el espectro de actividad de la población. Sin embargo, y a pesar del número de individuos avistados y el tiempo de trabajo de campo invertido en los dos estudios, no se observó ningún comportamiento reproductivo diferente del canto de los machos. No encontramos parejas amplexantes; en 2008-2009 se encontró una sola postura en el mes de octubre, mientras que en el trabajo realizado durante 2010-2011 no encontramos posturas.

G. walkeri tiene un repertorio vocal simple constituido por un solo tipo de llamada, con una única nota. La llamada tiene una duración de (Media $\pm \mathrm{DE}) 1.4 \pm 0.62 \mathrm{~s}, \quad(\mathrm{n}=5)$, constituida por siete notas repetidas, cada una con una duración de $0.062 \pm 0.019 \mathrm{~s}(\mathrm{n}=22)$ y un intervalo de $0.151 \pm 0.016 \mathrm{~s}(\mathrm{n}=7)$. El promedio de la frecuencia dominante es de $2544.73 \pm 119.69 \mathrm{~Hz}$ (Fig. 3).

Las condiciones del ambiente de los microhábitats donde se encontraron las ranas dentro de la hojarasca de pino se observan en el cuadro 2. Las tres variables están relacionadas con el régimen de precipitación del área (Regresión múltiple $\mathrm{R}=0.52, \mathrm{~F}=57.01, \mathrm{p}<0.001$ ) de manera que los valores promedio más bajos de humedad relativa y cobertura vegetal se observan durante los meses más secos, mientras que la

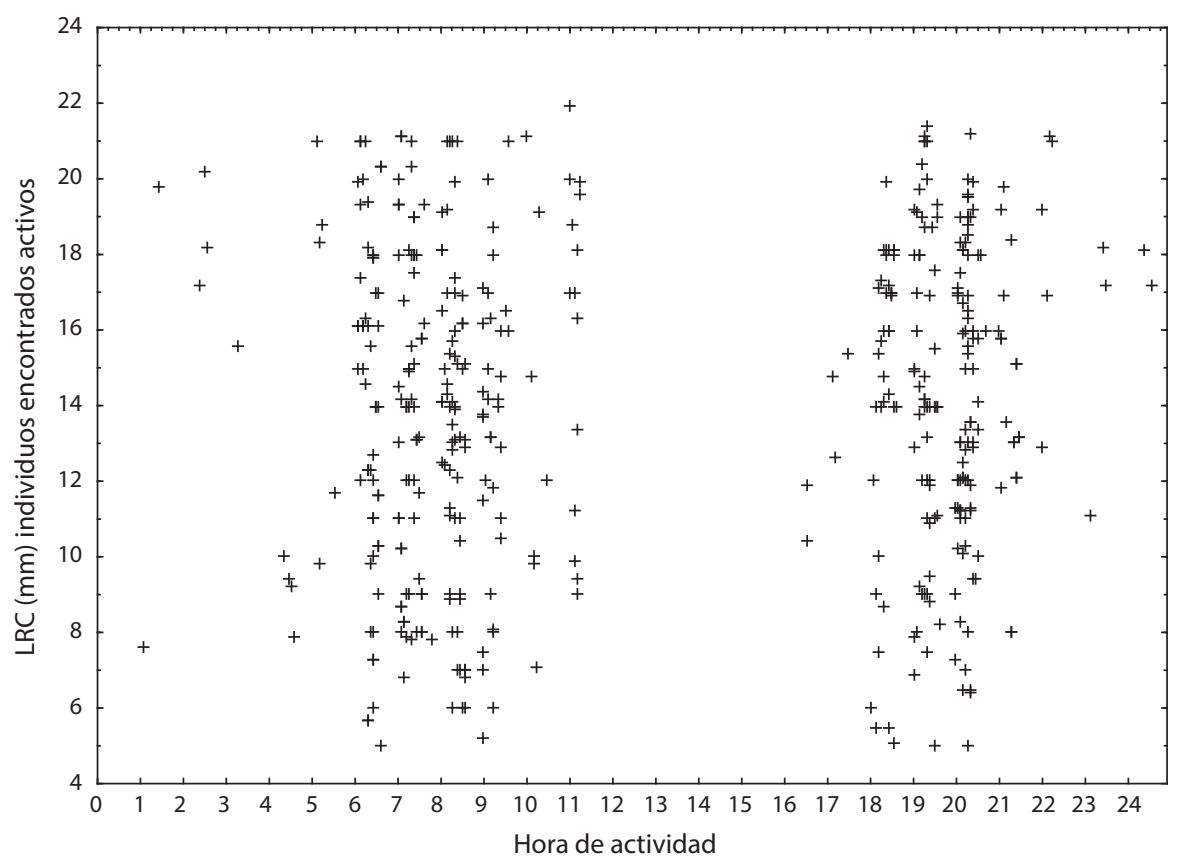

Fig. 2. Actividad diurna y nocturna de Geobatrachus walkeri en relación a su tamaño corporal. Los individuos encontrados activos (moviéndose, forrajeando, cantando) se encontraron durante horas diurnas y nocturnas y no hay diferencia entre la hora de actividad y el tamaño corporal o categoría de edad (adulto o juvenil).

Fig. 2. Diurnal and nocturnal activity in Geobatrachus walkeri related to its body size. Active individuals (in movement, foraging, calling) were found during day and night hours; there were no differences between the activity time and body size, and age category (adult or juvenile). 


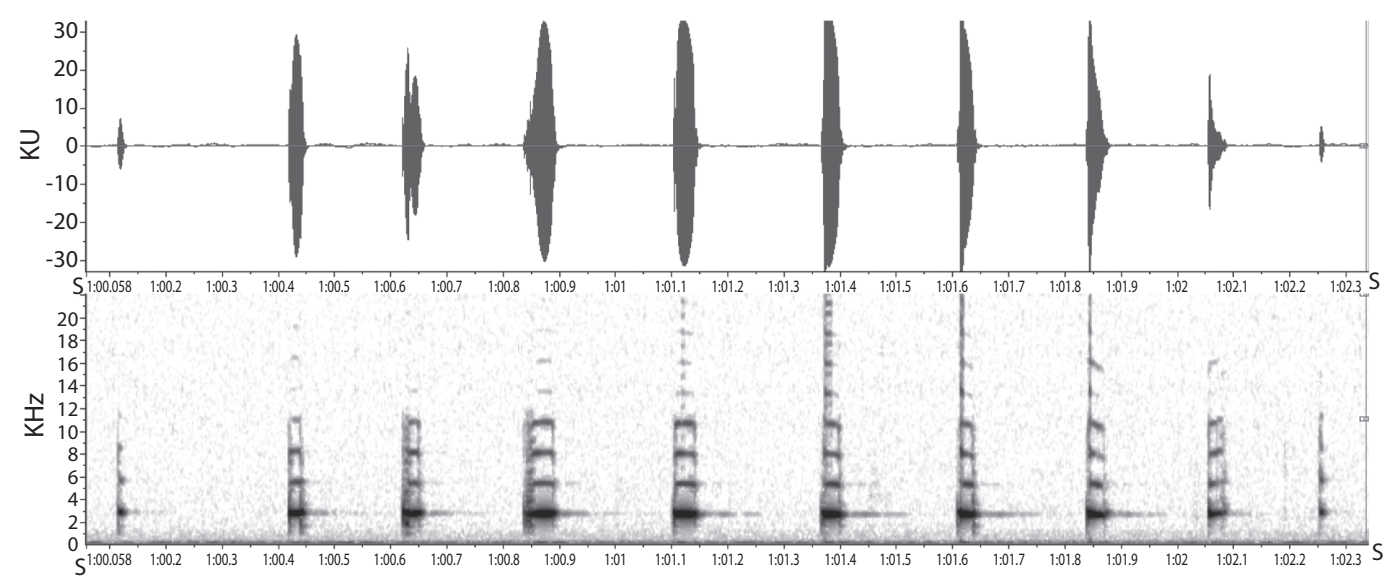

Fig. 3. Oscilograma (arriba, amplitud relativa en kU vs. tiempo) y sonograma (debajo, frecuencia en kHz vs. tiempo) del canto de Geobatrachus walkeri.

Fig. 3. Oscilogram (top, relative amplitude in $\mathrm{kU}$ vs. time) and sonogram (bottom, frequency in $\mathrm{kHz} v \mathrm{vs}$. time) of the calling of Geobatrachus walkeri.

CUADRO 2

Comparación de la variación entre meses y entre estaciones de las condiciones registradas de los microambientes donde se encontraron las ranas Geobatrachus walkeri en las dos temporadas de estudio

TABLE 2

Comparison of the intra-month and intra-season variation in the registered environmental conditions of the microhabitats of Geobatrachus walkeri in the two study years

\begin{tabular}{|c|c|c|c|c|c|}
\hline \multirow{2}{*}{$\begin{array}{l}\text { Condiciones del } \\
\text { ambiente }\end{array}$} & \multicolumn{2}{|c|}{$2008-2009$} & \multicolumn{3}{|c|}{$2010-2011$} \\
\hline & Promedio y rango & entre estaciones & Promedio y rango & entre meses & entre estaciones \\
\hline Humedad relativa & $\begin{array}{l}83.5 \pm 15 \\
(79-92 \%)\end{array}$ & NS & $\begin{array}{c}63.8 \pm 13.67 \\
(35-93 \%)\end{array}$ & $\begin{array}{c}\mathrm{H}=53.16, \mathrm{p}<\mathbf{0 . 0 0 0 1} \\
\text { Dic, Ene menor }\end{array}$ & NS \\
\hline Temperatura & $\begin{array}{l}14.4 \pm 1.5 \\
\left(10-20^{\circ} \mathrm{C}\right)\end{array}$ & $\begin{array}{c}\mathrm{H}=15.78, \mathrm{p}<\mathbf{0 . 0 0 1} \\
\quad \text { Seca menor }\end{array}$ & $\begin{array}{l}11.75 \pm 1.79 \\
\left(8.3-15.6^{\circ} \mathrm{C}\right)\end{array}$ & $\begin{array}{c}\mathrm{H}=25.4, \mathrm{p}<\mathbf{0 . 0 0 1} \\
\text { May mayor }\end{array}$ & NS \\
\hline Cobertura vegetal & $\begin{array}{c}79 \pm 7 \% \\
(13-86 \%)\end{array}$ & NS & $\begin{array}{c}63.4 \pm 14.9 \\
(29-98 \%)\end{array}$ & $\begin{array}{c}\mathrm{H}=20.7, \mathrm{p}=\mathbf{0 . 0 0 4} \\
\text { Jul mayor }\end{array}$ & $\begin{array}{c}H=5.9, p=\mathbf{0 . 0 1} \\
\text { Seca menor }\end{array}$ \\
\hline
\end{tabular}

relación es inversa con respecto de la temperatura, la cual es menor durante los meses de la estación de lluvias.

De la muestra de 42 ranas recolectadas 13 se identificaron como machos (12 adultos y un subadulto), 29 como hembras (28 adultas y una juvenil). El tamaño mínimo de madurez sexual fue $17.8 \mathrm{~mm}$ LRC para las hembras y $18.1 \mathrm{~mm}$ LRC en los machos. El tamaño promedio de los machos adultos fue de $20.5 \pm 0.75 \mathrm{~mm}$ y el de las hembras de $23.4 \pm 1.29 \mathrm{~mm}$. Comparando el tamaño corporal de los machos y hembras adultas se observa que aunque las hembras tienden a ser más grandes no se encontraron diferencias significativas ni en LRC ni en peso (Mann-Withney $\mathrm{U}=132$, $\mathrm{p}=0.3 \mathrm{y} \mathrm{U}=161$, $\mathrm{p}=0.7$, respectivamente).

Todas las hembras adultas recolectadas fueron reproductivas (estado 3), por lo tanto el estado reproductivo de las hembras no cambió significativamente entre meses o entre estaciones. A nivel histológico, 24 de las hembras presentaban folículos en estados V y VI (vitelogénesis tardía) y cuatro hembras encontradas en los meses de julio, agosto, octubre y diciembre, tenían folículos entre los estados del I a VI 
(inicio del crecimiento folicular a vitelogénesis tardía) y oviductos muy flácidos que sugieren una oviposición pasada reciente y un nuevo ciclo de crecimiento folicular. El número promedio de ovocitos en crecimiento simultáneo por hembra fue de $19.1 \pm 5.1$, este número no está correlacionado con el LRC de la hembra $\left(\mathrm{r}^{2}=0.17, \mathrm{p}=0.38\right)$; el diámetro promedio de los folículos yemados más avanzados en cada hembra fue de $1.81 \mathrm{~mm} \pm 0.42 \mathrm{~mm}$ y tampoco se correlacionó con el LRC de la hembra $\left(\mathrm{r}^{2}=0.25\right.$, $\mathrm{p}=0.2$ ). El diámetro folicular no cambió significativamente entre los meses de lluvias y $\operatorname{secos}(\mathrm{H}=0.71, \mathrm{p}=0.4)$, ni se relacionó con la precipitación mensual $\left(\mathrm{r}^{2}=-0.24, \mathrm{p}=0.22\right)$; sin embargo, los folículos más grandes que están cerca del tamaño de ovulación se observaron en octubre y enero (Fig. 4).

Los testículos de G. walkeri son pequeños (aproximadamente de $3.5 \times 1.5 \mathrm{~mm}$ de diámetro mayor y menor, respectivamente). La túnica albugínea siempre es pigmentada. No existieron diferencias significativas entre el volumen de los dos testículos ( $\mathrm{t}=0.95, \mathrm{gl}=11, \mathrm{p}=0.36)$, se empleó el testículo izquierdo para los análisis histológicos y de cambios del volumen entre estaciones. No se encontró una relación significativa entre el volumen testicular y la LCR $(\mathrm{r}=0.74, \mathrm{p}=0.41, \mathrm{n}=12)$. El volumen testicular cambió significativamente entre estaciones $(\mathrm{H}=4.15, \mathrm{p}=0.04)$, siendo significativamente mayor en los meses de la estación seca, y se relacionó significativa e inversamente con la precipitación $\left(\mathrm{r}^{2}=-0.43, \mathrm{p}=0.038\right)$. Todos los machos adultos examinados tenían espermatozoides libres en el lumen de los túbulos seminíferos. A pesar de que las observaciones de machos cantando durante todos los muestreos y los histológicos demuestran que los machos fueron reproductivos durante todos los

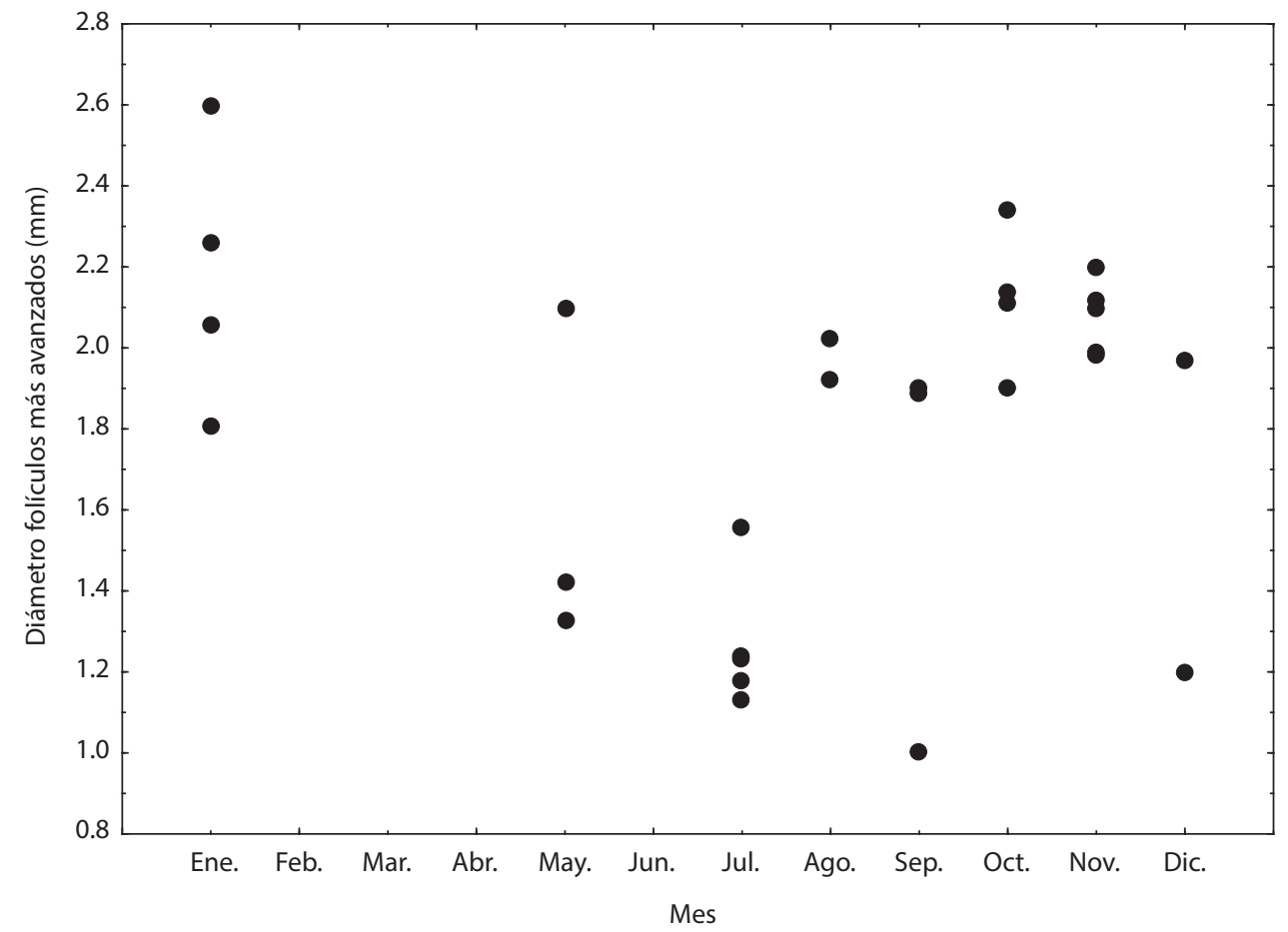

Fig. 4. Diámetro de los folículos ováricos más avanzados encontrados por hembra y mes de captura en Geobatrachus walkeri. Los folículos yemados se encuentran en hembras de todos los meses muestreados.

Fig. 4. Diameter of the advanced ovarian follicles in each female by month of capture in Geobatrachus walkeri. Females with vitellogenic follicles were found in all of the sampled months. 
muestreos, las proporciones relativas de cada tipo de cistos del proceso de la espermatogénesis y la cantidad de espermatozoides luminales se observaron variar. En diciembre-enero se vieron individuos con abundante esperma luminal y pocos cistos de espermatogonias y espermatocitos primarios, mientras que los demás meses se observaron cortes con abundantes espermatozoides pero una mayor proporción de epitelio espermático conformado por cistos en todos los estadios de la espermatogénesis; estas diferencias no parecen estar relacionadas con el tamaño corporal de los machos (Fig. 5). A pesar de que el número de machos estudiados
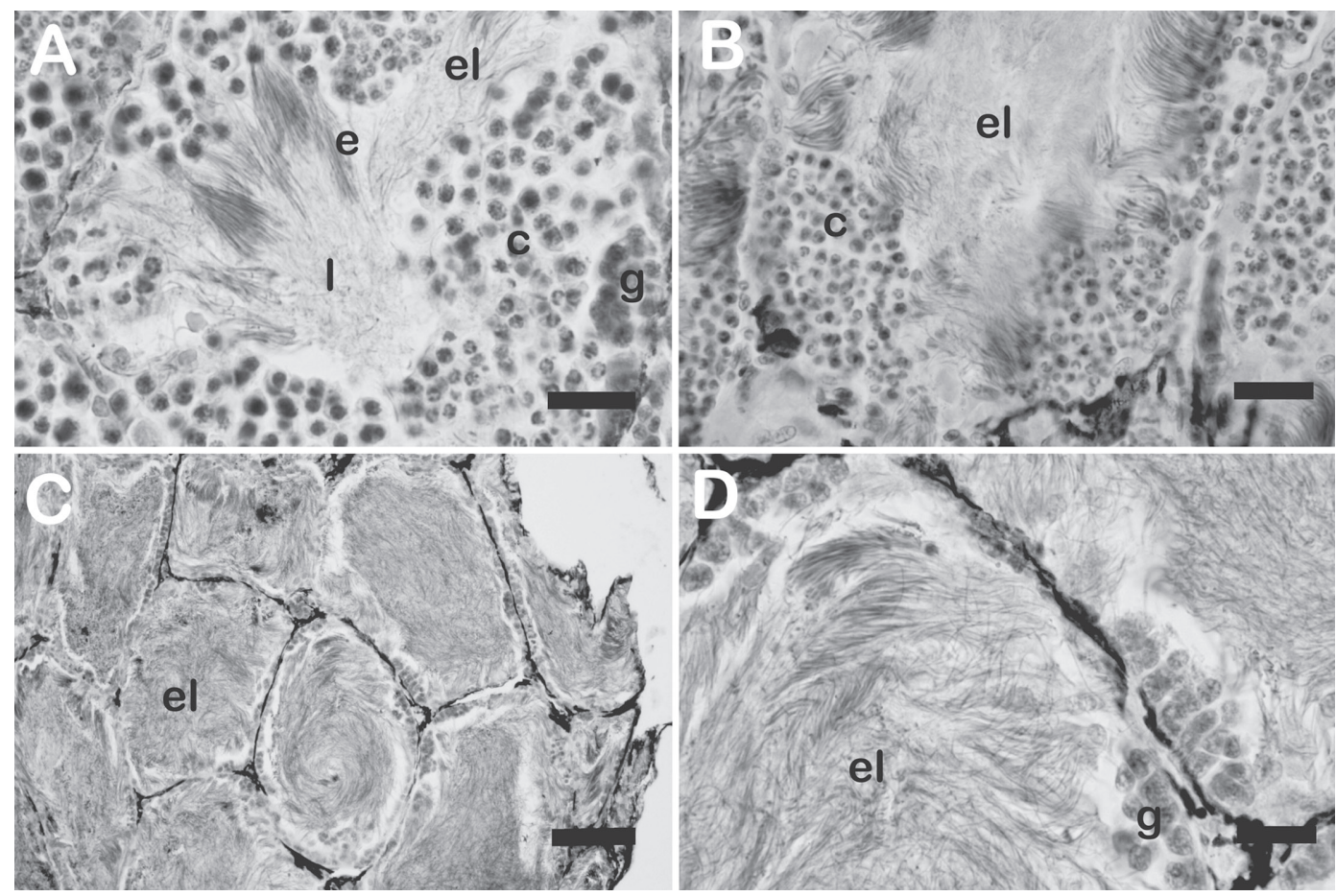

Fig. 5. Cortes transversales de testículos de Geobatrachus walkeri. Nótese que los túbulos seminíferos tienen una capa de células pigmentarias en el tejido conectivo que permite delimitarlos fácilmente. (A) Túbulo seminífero de un macho de mayo. El epitelio seminífero es amplio con cistos de toda la línea espermática y pocos espermatozoides en mechones y libres en la luz del túbulo. (B) Túbulo seminífero de un macho del mes de octubre con abundantes espermátides tardías, abundantes mechones de espermatozoides y muy abundante esperma luminal. (C) Panorámica de los túbulos seminíferos de un macho del mes de diciembre mostrando la gran cantidad de espermatozoides libres y en paquetes en la luz de los túbulos seminíferos. (D). Un detalle de los túbulos de este mismo macho muestra que el epitelio espermático es reducido a pocos cistos de espermatogonias, el resto del túbulo está completamente lleno de espermatozoides libres. c, cistos de espermatocitos; e, espermatozoides en mechones; el, espermatozoides libres en el lúmen tubular; g, cistos de espermatogonias; 1, lumen del túbulo seminífero.(A) Barra $=25 \mu \mathrm{m}, \mathbf{( B )}$ Barra $=30 \mu \mathrm{m}, \mathbf{( C ) B a r r a}=120 \mu \mathrm{m}, \mathbf{( D )}$ Barra $=15 \mu \mathrm{m}$.

Fig. 5. Transversal sections of the testes of Geobatrachus walkeri. The seminiferous tubules have a layer of pigment cells in the connective tissue that allows an easy delimitation of the tubules. (A) Seminiferous tubule of a male captured in May. The seminiferous epithelium is wide, containing all kind of cysts, few spermatozoa in bundles and free sperm at the lumen of the tubules. (B) Seminiferous tubule of a male captured in October with abundant late spermatids, abundant bundles of spermatozoa, and very abundant luminal sperm. (C) Panoramic of the seminiferous tubules of a male captured in December. It is observed a great quantity of bundles of spermatozoa and free sperm in the lumen of the tubules. (D) A detail of the same image shows that the spermatic epithelium is highly reduced to few cysts of spermatogonia, the rest of the tubule is completely full of spermatozoa. c, cysts of spermatocytes; e, spermatozoa in bundles; el, free spermatozoa in the lumen of the seminiferous tubules; g, cysts of spermatogonia; 1, lumen of the seminiferous tubule.(A) Scale bar $=25 \mu \mathrm{m}$, (B) Scale bar $=30 \mu \mathrm{m}$, (C) Scale bar $=120 \mu \mathrm{m}$, (D) Scale bar $=15 \mu \mathrm{m}$. 
a nivel histológico por mes es bajo, se observa un alto grado de sincronía en el estado de la espermatogénesis y apariencia de los túbulos seminíferos entre individuos del mismo mes de recolecta; así por ejemplo ocurre con los tres individuos estudiados en el mes de agosto (estación de lluvias) y en los 4 machos (3 del mes de diciembre) de los meses analizados de la estación seca.

\section{DISCUSIÓN}

En el presente estudio se confirma la observación previa de que la plantación de pino existente en la zona de estudio ofrece a $G$. walkeri microhábitats propicios para su establecimiento exitoso, siendo la hojarasca de pino utilizada ampliamente por esta especie tanto durante su actividad diaria como para refugiarse. Aunque el esfuerzo y tiempos de muestreo entre este estudio y el estudio pasado (Martínez-Baños et al., 2011) son diferentes, llama la atención el hecho de que en el presente estudio sólo se encontraron cuatro ranas en el bosque nativo en contraste con las 153 ranas encontradas en la plantación de pino, lo que hace la desproporción en la abundancia de ranas entre los dos hábitats mucho mayor a la reconocida hasta ahora. Este hecho y la reciente ampliación de la distribución de la especie al ecosistema páramo en la Sierra Nevada de Santa Marta (RuedaSolano \& Vargas-Salinas, 2010) en donde se localizaron especímenes en un ambiente muy diferente y utilizando microhábitats igualmente diferentes de los originalmente descritos para la especie (Ardila-Robayo, 1979) nos sugiere que esta especie tiene la capacidad de adaptarse a condiciones muy variadas dentro de su pequeño rango de distribución, demostrando plasticidad y ampliando así las posibilidades y oportunidades para su conservación.

Encontramos animales activos durante todos los muestreos en los dos años estudiados, pero en 2010-2011 se encontraron significativamente más ranas en uno de los meses de la estación seca (enero). Esta variación entre muestreos y el particular incremento en la abundancia de ranas en enero están relacionados con el incremento en la cantidad de juveniles en ese mes ya que la distribución temporal de los individuos de la población demostró que hubo diferencias significativas en el encuentro de juveniles, mientras que la cantidad de adultos encontrada se mantuvo. Así mismo, la categoría juveniles fue la más abundante y representada por un más amplio rango de tamaños corporales. El estudio sobre esta misma población (Martínez-Baños et al., 2011) no encontró cambios significativos en el número de ranas capturadas entre los meses de lluvias y secos; sin embargo, a diferencia del presente estudio, la mayor abundancia de ranas se registró en la estación de lluvias.

Estos resultados muestran que puede haber períodos particulares de reclutamiento de juveniles y que el periodo de crecimiento para alcanzar la talla de madurez es relativamente largo. En el estudio de 2010-2011 los juveniles más pequeños observados se encontraron en la estación seca por lo tanto se puede pensar que los nacimientos pudieron ocurrir en la transición entre la estación de lluvias e inicio de la estación seca. Hay sin embargo también juveniles de tallas pequeñas en septiembre y noviembre lo que indicaría que hay otros momentos de reclutamiento de juveniles durante la prolongada estación de lluvias. Asimismo, en 2008-2009 se encontraron juveniles de tallas cercanas a la eclosión (menos de $6 \mathrm{~mm}$ de LRC) en los meses de julio y octubre, meses de la estación de lluvias, lo que apoyaría la idea de que no hay un único período de reclutamiento de juveniles, sino que este puede ocurrir durante toda la estación de lluvias, con aparentemente un pico en la transición entre la estación de lluvias y la estación seca.

Watling and Donelly (2002) en Costa Rica encontraron también un incremento en la abundancia de ranas de hojarasca durante la estación seca debido al incremento en el número de juveniles durante esta época. Con base en sus resultados y la revisión de los patrones de abundancia de ranas de hojarasca en el Neotrópico estos autores sugieren que los patrones de abundancia entre Centro América y Sur América difieren de manera que los juveniles son 
reclutados predominantemente en la estación seca en Centro América, mientras que el reclutamiento de juveniles ocurre en la estación de lluvias en Sur América. Los autores atribuyen estas diferencias a los diferentes patrones de abundancia de artrópodos presa entre ambas áreas geográficas. Para el caso de G. walkeri aunque hay un pico claro de aparición en los muestreos de juveniles de menor tamaño corporal en enero, un mes de mínima precipitación, se encuentran también posturas al inicio de la estación de lluvias (abril y mayo, ArdilaRobayo, 1979), durante la estación de lluvias en octubre (Martínez-Baños et al., 2011), y juveniles de tamaños mínimos en varios meses de la estación de lluvias. Por lo tanto, aunque hay algún grado de estacionalidad y sincronía en la abundancia de juveniles en esta población, el reclutamiento de juveniles no es exclusivo de ninguna de las dos estaciones y sugiere entonces una actividad reproductiva continua con alguna variación. Este resultado permite inferir que hay recursos suficientes para la reproducción durante ambas estaciones y que algún factor puede estar relacionado con una mayor o menor tasa reproductiva en el tiempo. Si de acuerdo con la hipótesis de Watling \& Donelly (2002) es la disponibilidad de presas para los adultos que se reproducen o para los neonatos en crecimiento el factor que determina esta fluctuación, debería encontrarse una disponibilidad de presas permanente durante las dos estaciones y significativamente mayor al inicio o durante la estación seca en este ambiente. Un análisis detallado de la dieta y la disponibilidad de presas en el área permitiría probar esta hipótesis para esta población.

Para especies del clado Terrarana, como en $G$. walkeri, se ha encontrado también que es común encontrar una gran cantidad de juveniles con relación a la proporción de adultos (p. ej. Pristimantis douglasi, P. merostictus, P. miyatai, Arroyo, Serrano-Cardozo \& Ramírez-Pinilla, 2008; Craugastor bransfordii Donnelly, 1999; Eleutherodactylus johnstonei, Ovaska, 1991), por lo que se sugiere que conforman poblaciones estables (Stearns, 1976). Para el caso de $G$. walkeri se encontraron juveniles de todas las tallas en los diferentes meses muestreados lo que indicaría que representan varias cohortes de juveniles que crecen simultáneamente y fueron reclutados durante los varios eventos que se observan en el año.

$G$. walkeri tiene actividad diurna y nocturna con un pico de actividad entre las 20 y $21 \mathrm{~h}$ para todas las clases de edad. Este patrón de actividad durante todo el día con picos de actividad nocturnos no es común en anuros, que suelen ser asociados con actividad nocturna (Duellman \& Trueb, 1994); sin embargo, otras especies de ranas de hojarasca y del clado Terrarana tienen este mismo patrón (e.j. Pristimantis douglasi Arroyo et al., 2008, P.aff. fenestratus, Waldez, Menin, \& Rojas-Ahumada, 2011). Para comunidades de ranas de hojarasca de bosque, se sugiere que la actividad diurna es preponderante (Lima \& Moreira, 1993); sin embargo, se ha observado que el método de muestreo con el que se ha establecido la actividad en comunidades de ranas características de este microhábitat (cuadrantes diurnos) sesga los patrones de actividad y que haciendo simultáneamente cuadrantes nocturnos y diurnos se puede ver que las ranas de hojarasca tienen mayor actividad nocturna que diurna (Rocha,Van Sluys, Alves, Bergallo \& Vrcibradic, 2000) como es en el caso de $G$. walkeri. De otro lado, a diferencia de varias especies de Pristimantis no hay segregación entre categorías de edad en la actividad diaria, los juveniles de G. walkeri son activos simultáneamente con los adultos durante todo el rango de actividad observado en la población. Para las especies donde se ha visto esta segregación temporal parece haber también una segregación espacial de manera que mientras los juveniles son terrestres y diurnos, los adultos usualmente perchan a varias alturas sobre la vegetación y son activos durante la noche, cuando cantan y forrajean (p.ej. P. aff. fenestratus Waldez et al., 2011). Para el caso de G. walkeri no observamos individuos perchando sobre la vegetación arbustiva en este ambiente de manera que adultos y juveniles no están segregados ni espacial ni temporalmente. 
La actividad observada para esta población, que incluyó machos cantando, individuos caminando y forrajeando, se observó siempre entre o sobre la hojarasca de la plantación de pino pero nunca sobre la vegetación herbácea o arbustiva por encima del suelo, lo que sugiere que el microhábitat de esta población es terrestre y en este caso asociado a la hojarasca del pino o bajo las piedras en el bosque nativo. Sin embargo, de manera interesante en el ecosistema páramo (ca. de $1000 \mathrm{~m}$ más de altitud que en nuestro sitio de trabajo) Rueda-Solano \& Vargas-Salinas (2010) encontraron durante el día machos cantando sobre montículos de vegetación muy cercanos a los cursos de agua en la zona, un microhábitat no descrito hasta el momento para la especie.

Las condiciones observadas de los microhábitats donde se hallaron los individuos en los dos años de muestreo respecto de las variables registradas son diferentes en promedio y rango; en general los valores de humedad relativa, temperatura del suelo y cobertura vegetal fueron mayores en el año 2008-2009 respecto a los registrados en el trabajo de campo de 20102011. De manera coincidente ambos periodos de estudio correspondieron a años del fenómeno de La Niña, siendo el más reciente mucho más fuerte. En 2007 se inició La Niña y se prolongó hasta 2009; sin embargo, aunque prolongado, no se consideró de intensidad fuerte. Para 2010 el mes de julio fue el más lluvioso de los últimos 40 años en la región Caribe y Andina colombianas, así como también fueron mucho mayores las lluvias hasta finalizar el año (IDEAM, 2011).

Todas las hembras y los machos por encima de $18 \mathrm{~mm}$ de LRC fueron reproductivos en los meses muestreados, lo que implica que la reproducción es potencialmente continua en esta especie. Las hembras tuvieron folículos vitelogénicos avanzados durante todos los meses e incluso algunas hembras fueron simultáneamente postgrávidas y vitelogénicas lo que sugiere que una vez se da la oviposición un nuevo ciclo de crecimiento folicular puede iniciar inmediatamente y que las hembras pueden reproducirse varias veces por año. En el caso de los machos, estos fueron así mismo reproductivos todos los meses muestreados; sin embargo, se observó claramente variación en esta actividad especialmente en los meses de diciembre-enero en los que se observó un significativo mayor volumen testicular y una masiva liberación de espermatozoides y una disminución drástica de los demás elementos del epitelio espermático, quedando solamente cistos de espermatogonias y espermatocitos primarios. Esta condición testicular puede sugerir que la espermatogénesis final puede tener un receso temporal durante los meses posteriores en la temporada seca. Infortunadamente por razones logísticas no hemos podido hacer muestreos en los meses posteriores de la estación seca (febrero a marzo) ni durante el inicio de la temporada de lluvias en abril; es posible que para estos meses la actividad testicular muestre sólo los primeros eventos del proceso espermatogénico y los machos no sean efectivamente reproductivos. Así, la drasticidad de la estación seca podría parar o disminuir la actividad reproductiva; sólo un estudio que involucre recolectas durante estos meses puede comprobar esta hipótesis.

Inferimos el tamaño de postura en $G$. walkeri por el número de folículos avanzados que crecen sincrónicamente; no encontramos sin embargo en este trabajo ninguna postura en el campo. En 2008-2009 Martínez-Baños et al. (2011) registraron el hallazgo de una postura en octubre entre los refugios de la hojarasca de pino demostrando que al menos la oviposición sí puede darse en este microambiente. El tamaño de esta postura registrada fue de 23 huevos, que está dentro del rango del conteo folicular del presente trabajo. Es común en anuros encontrar una correlación significativa entre el tamaño de la hembra y el tamaño de la postura (Salthe \& Duellman, 1973).En G. walkeri no encontramos esta relación (con relación al número de folículos vitelogénicos en desarrollo simultáneo), aunque es esperable dado que el número de hembras analizadas es pequeño y el rango de variación del tamaño en LRC de las hembras es estrecho. Dentro de Terrarana algunas especies han demostrado esta relación 
con respecto al número de huevos (p. ej. Pristimantis altamazonicus, $P$. lacrimosus, $P$. lanthanites, Crump, 1974; C. bransfordii, Donnelly, 1999; Ischnocnema parva Martins et al., 2010; E. johnstonei Ortega et al., 2005; E. coqui, Townsend \& Stewart 1994) o al tamaño de los huevos (p. ej. Brachycephalus didactylus, Almeida-Santos, Siqueira, Van Sluys \& Rocha, 2011), pero otras varias especies como en $G$. walkeri no demuestran esta relación (p. ej. Pristimantis conspicillatus, P. croceoinguinis, P. martiae, Oreobates quixensis, Crump, 1974).

De acuerdo con los resultados obtenidos en $G$. walkeri podemos pensar que la población estudiada puede reproducirse continuamente durante la temporada de lluvias con picos de reclutamiento de juveniles al final de esta estación e inicio de la temporada seca. Sin embargo, no sabemos aún si la intensidad de la estación seca induzca un cese completo de la actividad reproductiva, ya sea por estacionalidad en la actividad gonadal como lo sugiere el análisis histológico testicular o por una disminución en la oviposición o en el éxito del desarrollo de las posturas, lo que determinaría una actividad reproductiva estacional.

\section{AGRADECIMIENTOS}

Nuestro agradecimiento al señor Jorge Torregrosa por su colaboración en el área de estudio. A Víctor Hugo Serrano y Juan Pablo Ramírez por sus aportes y comentarios al documento; a Gustavo Rúa y Arístides López quienes colaboraron en algunas de las fases del trabajo de campo, a Vera Martínez quien organizó y aportó los datos del primer estudio realizado en 2008-2009, a Jesús E. Ortega por su colaboración en el análisis del canto y a Eliana P. Ramos quien colaboró en el trabajo histológico. La Unidad Administrativa Especial del Sistema de Parques Nacionales-Sierra Nevada de Santa Marta y CORPAMAG otorgaron los permisos de recolecta e investigación. Colciencias, la Universidad del Magdalena y la Universidad Industrial de Santander apoyaron a la primera autora con el otorgamiento del estímulo como joven investigadora.

\section{RESUMEN}

Geobatrachus walkeri es una rana que pertenece a un género monotípico y es endémica de la Sierra Nevada de Santa Marta, Colombia. La especie habita en diferentes microhábitats entre los 2000 y $3500 \mathrm{~m}$ de altitud, incluyendo la hojarasca de una plantación de pinos. Para entender su ecología reproductiva se realizaron ocho muestreos de individuos, que abarcaron las estaciones de lluvias y de sequía y dos tipos de hábitats (bosque secundario y plantación de pino) durante 2010-2011. También se incluyeron los datos de cinco muestreos similares obtenidos previamente durante el período 2008-2009. El principal microhábitat donde las ranas se encontraron fue la hojarasca de pino, allí escuchamos coros de seis-diez machos durante todos los meses muestreados, y observamos que las ranas tienen actividad diurna y nocturna. Sin importar el año de estudio, la población se compone de neonatos reclutados en varios meses del año, un gran número de juveniles con un amplio rango de tamaños corporales, y un menor número de adultos con un rango más reducido de tamaño corporal. Los análisis histológicos de las gónadas mostraron que el tamaño a la madurez se adquiere cerca de los $18 \mathrm{~mm}$ LRC para machos y hembras, y que los machos y hembras adultos fueron reproductivos durante todo el muestreo, lo que sugiere un patrón continuo de actividad reproductiva. Sin embargo, durante la estación seca, los túbulos seminíferos presentan un epitelio espermático drásticamente disminuido, aunque contienen abundantes espermatozoides luminales, lo que sugiere una reducción en la producción de espermatozoides en el final de la estación seca. Del mismo modo, las ranas de todas las categorías de edad fueron significativamente más abundantes durante el inicio de la estación seca, mientras que fueron significativamente menos abundantes con el avance de la sequedad, lo que sugiere que una mayor intensidad de la temporada de sequía podría detener temporalmente la actividad y la reproducción en esta población.

Palabras clave: actividad reproductiva, Sierra Nevada de Santa Marta, estacionalidad tropical, rana de hojarasca de montaña, histología gonadal.

\section{REFERENCIAS}

Almeida-Santos, M., Siqueira, C. C., Van Sluys, M., \& Rocha, C. F. D. (2011). Ecology of the Brazilian flea frog Brachycephalus didactylus (Terrarana: Brachycephalidae). Journal of Herpetology, 45, 251-255.

Ardila-Robayo, M. C. (1979). Status sistemático del género Geobatrachus Ruthven 1915 (Amphibia: Anura). Caldasia, 12, 383-496.

Arroyo, S. B., Serrano-Cardozo, V. H., \& Ramírez-Pinilla, M. P. (2008). Diet, microhabitat and time of activity in a Pristimantis (Anura, Strabomantidae) assemblage. Phyllomedusa, 7, 10-119. 
Bolaños, F. (2003). Ciclos de vida de los anfibios, con énfasis en Costa Rica. In R. Acuña-Mesén (Ed.). La Biodiversidad (p. 89-108). San José. Costa Rica: Editorial de la Universidad de Costa Rica.

Bourne, G. R. (1997).Reproductive behavior of terrestrial breeding frogs Eleutherodactylus johnstonei in Guyana. Journal of Herpetology, 31, 221-229.

Canedo, C. \& Rickli, E. (2006). Female reproductive aspects and seasonality in the reproduction of Eleutherodactylus binotatus (Spix, 1824) (Amphibia, Leptodactylidae) in an Atlantic rainforest fragment, Southeastern Brazil. Herpetological Review, 37, 149-151.

Charif, R. A, Ponirakis, D. W., \& Krein, T. P. (2008). Raven Lite 1.5 user's guide. Ithaca: Cornell Laboratory of Ornithology.

Cocroft, R. B. \& Ryan, M. J. (1995). Patterns of advertisement call evolution in toads and chorus frogs. Animal Behavior, 49, 283-303.

Crump, M. L. (1974). Reproductive strategies in a tropical anuran community. University of Kansas Museum of Natural History, Miscellaneous Publications, 61, 1-68.

Crump, M. L. (1982). Amphibian reproductive ecology in the community level. In N. J. Scott (Ed.), Herpetological Communities (p. 239). U.S. Department of the Interior Fishand Wildlife Service. Washington D.C.

Crump, M. L. \& Scott, N. J. (1994). Standard Techniques for inventory and monitoring. In R. W. Heyer, M. A. Donnelly, R. W. Mcdiarmid, L. A. Hayek, \& M. S. Foster (Eds.), Measuring and monitoring biological diversity. Standard Methods for Amphibians (p. 77-171). The Smithsonian Institution, Washington, D. C., EEUU.

Donnelly, M. A. (1994). Amphibian diversity and natural history. In L. McDade, K. Bawa, H. Hespenheide, \& G. Hartshorn (Eds.), La Selva: Ecology and Natural History of a Neotropical Rainforest (p. 199-209). Chicago: University of Chicago Press.

Donnelly, M. A. (1999). Reproductive phenology of Eleutherodactylus bransfordii in Northeastern Costa Rica. Journal of Herpetology, 33, 624-631.

Donnelly, M. A. \& Guyer, C. (1994). Patterns of reproduction and habitat use in anassemblage of Neotropical hylid frogs. Oecologia, 98, 291-302.

Duellman, W. E. \& Trueb, L. (1994). Biology of amphibians. Baltimore and London: The Johns Hopkins University Press.

Fong, A., Hero, A. J. M., Viña, R., \& Bignotte-Giró, I. (2010). Population ecology of the riparian frog Eleutherodactylus cuneatus in Cuba. Biotropica, 42, 348-354.

Guayasamin, J. M., Bonaccorso, E., Menendez, P. A., \& Bustamante, M. R. (2004). Morphological variation, diet, and vocalization of Eleutherodactylus eugeniae (Anura: Leptodactylidae) with notes on its reproduction and ecology. Herpetological Review, 35, 17-23.

Hedges, S. B., Duellman, W. E., \&. Heinicke, M. P. (2008). New World direct-developing frogs (Anura: Terrarana): Molecular phylogeny, classification, biogeography, and conservation. Zootaxa, 1737, 1-182.

IDEAM (2011). Boletín N ${ }^{\circ} 25$ sobre el monitoreo del Fenómeno de "La Niña" 20 de enero de 2011. (Recuperado enero 10 de 2013 http://www.pronosticosyalertas. gov.co/).

Lima, A. P., \& Moreira, G. (1993). Effects of prey size and foraging mode on the ontogenetic change in feeding niche of Colostethus stepheni (Anura: Dendrobatidae). Oecologia, 95, 93-102.

Marsh, D. M. \& Pearman, P. B. (1997). Effects of habitat fragmentation on the abundance of two species of Leptodactylid frogs in an Andean montane forest. Conservation Biology, 11, 1323-1328.

Martínez-Baños, V. T., Pacheco-Florez, V., \& RamírezPinilla, M. P. (2011). Abundancia relativa y uso de microhábitat de la rana Geobatrachus walkeri (Anura: Strabomantidae) en dos hábitats en Sierra Nevada de Santa Marta, Colombia. Revista de Biología Tropical, 59, 907-920.

Martins, A. C. J. S., Kiefer, M. C., Siqueira, C. C., Van Sluys, M., Menezes, V. A., \& Rocha, C. F. D. (2010). Ecology of Ischnocnema parva (Anura: Brachycephalidae) at the Atlantic Rainforest of Serra da Concórdia, state of Rio de Janeiro, Brazil. Zoologia, 27, 201-208.

Menin, M., Waldez, F., \& Lima, A. P. (2008). Temporal variation in the abundance and number of species of frogs in 10,000 ha of a forest in Central Amazonia, Brazil. South American Journal of Herpetology, 3, 68-81.

Ortega, J. E., Serrano, V. H., \& Ramírez-Pinilla, M. P. (2005). Reproduction of an introduced population of Eleutherodactylus johnstonei at Bucaramanga, Colombia. Copeia, 2005(3), 642-648.

Ovaska, K. (1991). Reproductive phenology, populationstructure, and habitat use of the frog Eleutherodactylus johnstonei in Barbados, West Indies. Journal of Herpetology, 25, 424-430.

Prado, C. P. A., Abdalla, F. C., Silva, A. P. Z., \& Zina. J.(2004).Late gametogenesis in Leptodactylus labyrinthicus (Amphibia, Anura, Leptodactylidae) and some ecological considerations. Brazilian Journal of Morphological Sciences, 21, 177-184.

Pough, F. H., Andrews, R. M., Cadle, J. E., Crump, M. L., Savitsky, A. H., \& Wells, K. D. (2004). Herpetology. Pearson Prentice Hall, Upper Saddle River, New Jersey. 
Ramírez-Pinilla, M. P., Osorno-Muñoz, M., Rueda, J. V., Amézquita, A., \& Ardila-Robayo, M. C. (2004). Geobatrachus walkeri. IUCN 2009. IUCN Red List of Threatened Species.Version 2009.1. (Recuperado 10 enero 2013, www.iucnredlist.org).

Rincón-Castro, F. \& Castro-Herrera, F. (1998). Aspectos ecológicos de una comunidad de Eleutherodactylus (Anura: Leptodactylidae) en un bosque de niebla del occidente de Colombia. Caldasia, 20, 193-202.

Rocha, C. F. D., Van Sluys, M., Alves, M. A. S., Bergallo, H. G., \& Vrcibradic, D. (2000). Activity of leaf-litter frogs: When should frogs be sampled? Journal of Herpetology, 34, 285-287.

Rogowitz, G. L., Candelaria, C. L., Denizard, L. E., \& Meléndez, L. J. (2001). Seasonal reproduction of a Neotropical frog, the cave coquí (Eleutherodactylus cooki). Copeia, 2001(2), 542-547.

Rueda-Solano, L. A. \& Vargas-Salinas, F. (2010). Amphibia, Anura, Strabomantidae, Geobatrachus walkeri (Ruthven, 1915): Altitudinal extension and new habitat, Colombia. Check List, 6, 454-455.

Salthe, S. N., \& Duellman, W. E. (1973). Quantitative constraints associated with reproductive mode in anurans. In J. L. Vial (Ed.) Evolutionary Biology of the Anurans. Contemporary Research on Major Problems (p. 229-249). Columbia, Missouri: University of Missouri Press.

Scott, N. J. (1976). The abundance and diversity of herpetofaunas of tropical forest litter. Biotropica, 8, 41-58.

Stearns, S. C. (1976). Life history tactics: A review of the ideas. Quarterly Review of Biology, 51, 3-47.
Tocher, M. D., Gascon, C., \& Zimmerman, B. (1997). Fragmentation effects on a central Amazonian frog community. A ten years study. In Laurance, W. \& Bierregaard, R. (Eds.). Tropical forest remnants (p. 124-137). Chicago, EEUU: Universidad de Chicago.

Towsend, D. S. \& Stewart, M. M. (1994).Reproductive ecology of the Puerto Rican frog Eleutherodactylus coqui. Journal of Herpetology, 28, 34-40.

Urbina-González, J. C. \& Galeano, S. (2009). Abundance, activity patterns and microhabitat of Rhinella macrorhina, an endemic toad from the cloud forests of the Colombian Central Andes. Herpetology Journal, 19, 35-40.

Waldez, F., Menin, M., Rojas-Ahumada, D. P., \& Lima, A. P. (2011). Population structure and reproductive pattern of Pristimantis aff. fenestratus (Anura: Strabomantidae) in two non-flooded forests of Central Amazonia, Brazil. South American Journal of Herpetology, 6, 119-126.

Watling, J. \& Donnelly, M. D. (2002). Seasonal patterns of reproduction and abundance of leaf litter frogs in a Central American rainforest. Journal of Zoology, 258, 269-276.

Wells, K. D. (2007). Ecology and Behavior of Amphibians. Chicago: University of Chicago Press.

Wiederhecker, H. C., Pinto, A. C. S., \& Colli, G. R. (2002). Reproductive ecology of Tropidurus torquatus (Squamata: Tropiduridae) in the highly seasonal Cerrado biome of Central Brazil. Journal of Herpetology, 36, 82-91.

Zar, H. H. (1999). Biostatistical Analysis. Nueva Jersey, EEUU: Prentice Hall, Englewood Cliffs. 
